\section{FROM WASHINGTON}

\section{NSF Notes \\ Integrated Sensors Research Center \\ Established}

The Integrated Sensors Center has been established at the University of CaliforniaBerkeley for research on integrated sensors. Directed by Richard M. White and Richard S. Muller, both professors of electrical engineering and computer sciences, the interdisciplinary center will involve faculty, students, and research fellows in electrical, mechanical, and chemical engineering; computer sciences; materials science; and other related fields.

Among the initial research projects at the center will be studies of sensor circuitry, materials and fabrication, and work on the use of ultrasound in sensors, optical sensing, and development of new sensing devices.

The Integrated Sensors Center is being initiated by a five-year continuing $\$ 450,000$ grant from NSF. The seed grant was awarded by NSF's Office of Cross Disciplinary Research as part of its program of Industry/University Cooperative Research Centers. Other funding will be provided by ind ustrial firms and organizations that will play an active role in the research. As many as a dozen member organizations will contribute $\$ 40,000$ a nnually to the center. The center is expected to become self-sustaining through its industrial membership within five years.

\section{Earthquake Engineering Research Center \\ Established}

NSF's National Science Board approved an award to the State University of New York (SUNY) at Buffalo to establish the first federally funded Earthquake Engineering Research Center in the United States.

Directed by Robert L. Ketter, Leading Professor of Engineering and Applied Sciences, the center will conduct research to improve basic knowledge about earthquakes, engineering practice, and procedures to minimize the loss of lives and property. Initial studies will be directed toward buildings and other structures built before the need to mitigate the effects of earthquakes was realized. Emphasis will be on integrating consideration of other hazards, such as high winds, with earthquake requirements.

Although SUNY-Buffalo will be the focal point, the center will involve the coordinated efforts of the City College of New York, Columbia University, Cornell University, Lamont-Doherty Geological Observatory, Lehigh University, Princeton University, and Rensselaer Polytechnic Institute.

In addition to their primary function, these institutions will develop graduate courses in earthquake hazard mitigation. Extensive technology transfer will be undertaken on a nationwide basis and coordinated with existing national activities.

\section{EDITOR'S CHOICE}

Figures appearing in the EDITOR'S CHOICE are those arising from materials research which strike the editor's fancy as being aesthetically appenling and eye-catching. No further criteria are applied and none should be assumed. Submissions of candidate figures are welcome and should include a complete source citation, a photocopy of the report in which it appears (or will appear), and a reproductionquality original drawing or photograph of the figure in question.

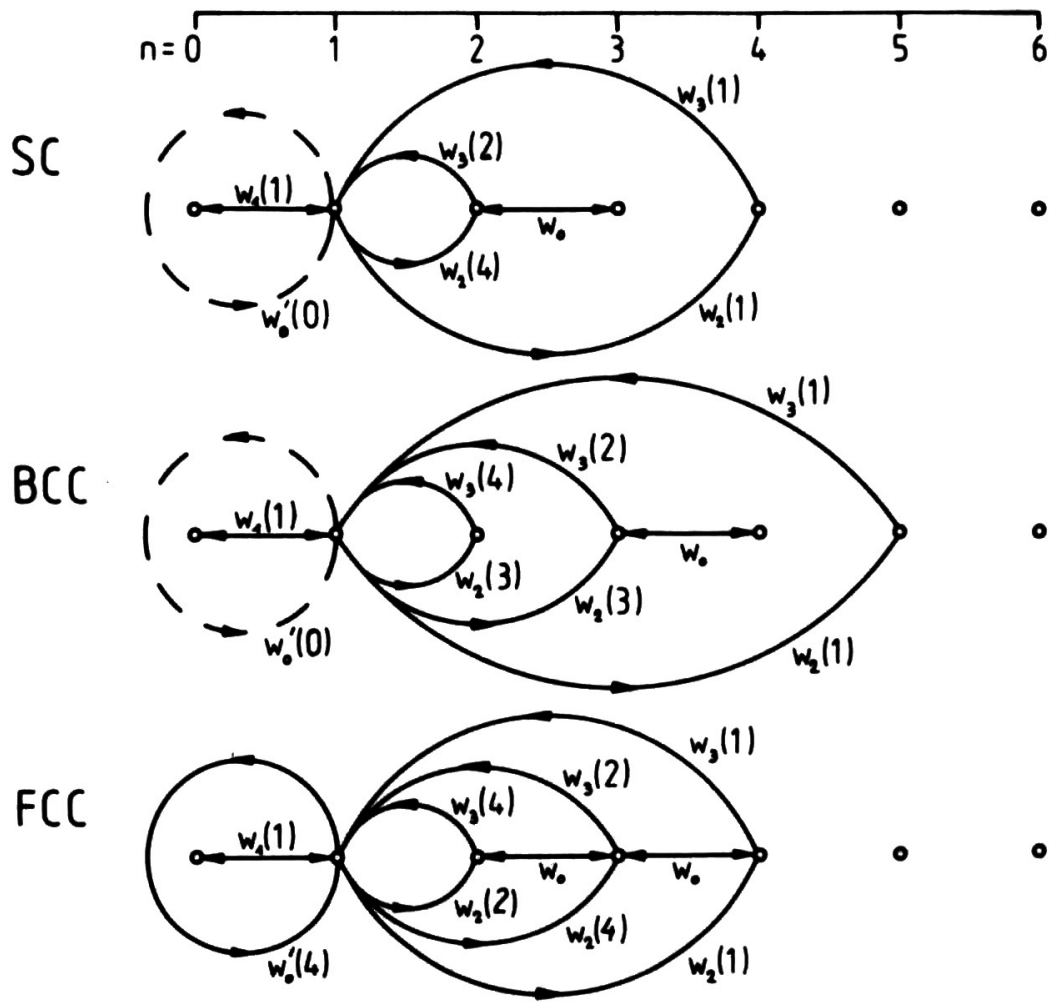

The EDITOR'S CHOICE for this issue of the BULLETIN comes from the work of K. Ruebenbauer and B. Sepiol of the Institute of Nuclear Physics, Kracow, Poland (Hyperfine Internctions 30, (1986) p. 121). In examining the impurity diffusion problem, the authors apply the encounter approximation to compute the diffusional selfcorrelation function. To derive the radial part of the function, they enumerate the various jumps that may occur between monovacancy and impurity to simple-cubic (sc), body-centered-cubic (bcc), and face-centered-cubic (fcc) lat tices and display the various paths schematically labeled by associated jump probabilities per unit time (w's). With some imagination, these blimp-like figures convey the illusion of motion as if to depict abstract aquatic life. 